Revue de droit comparé du travail et de la sécurité sociale

2 | 2020

La vie personnelle du salarié

\title{
La réglementation en matière de dépistage des drogues sur le lieu de travail en Nouvelle-Zélande
}

Peter Upson

\section{(2) OpenEdition}

1 Journals

Édition électronique

URL : https://journals.openedition.org/rdctss/1062

DOI : $10.4000 /$ rdctss. 1062

ISSN : 2262-9815

Éditeur

Centre de droit comparé du travail et de la sécurité sociale

Édition imprimée

Date de publication : 1 juin 2020

Pagination : 36-47

ISSN : 2117-4350

Référence électronique

Peter Upson, « La réglementation en matière de dépistage des drogues sur le lieu de travail en

Nouvelle-Zélande ", Revue de droit comparé du travail et de la sécurité sociale [En ligne], 2 | 2020, mis en ligne le 01 novembre 2021, consulté le 11 novembre 2021. URL : http://journals.openedition.org/ rdctss/1062; DOI : https://doi.org/10.4000/rdctss.1062

\section{c) (i) (5)}

Revue de droit comparé du travail et de la sécurité sociale est mise à disposition selon les termes de la Licence Creative Commons Attribution - Pas d'Utilisation Commerciale - Pas de Modification 4.0 International. 


\section{LA RÉGLEMENTATION EN MATIÈRE DE DÉPISTAGE DES DROGUES SUR LE LIEU DE TRAVAIL EN NOUVELLE-ZÉLANDE}

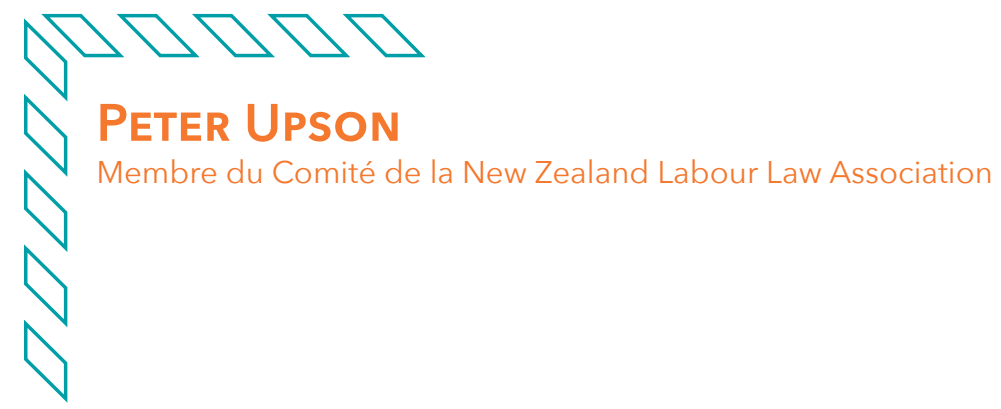

RÉSUMÉ

En Nouvelle-Zélande, la réglementation régissant le dépistage des drogues en milieu de travail est trop intrusive et empiète sur la vie privée des travailleurs. Dans certains secteurs spécifiques à haut risque, il est aujourd'hui possible de réaliser, de manière aléatoire, des tests de dépistage préalables à l'emploi, à la condition toutefois qu'il existe un motif raisonnable de soupçonner l'usage de drogues, ou à la suite d'un incident lié à la santé et à la sécurité. La New Zealand Drug Foundation a suggéré une réforme de la réglementation actuelle. Un examen de la jurisprudence en la matière semble étayer cette position.

Mots-clés : Dépistage des drogues sur le lieu de travail, vie privée, altération des facultés, santé et sécurité, libertés civiles

\section{ABSTRACT}

The regulations governing workplace drug testing are overly intrusive into the personal lives of workers. Currently drug testing can take place in pre-employment, at random in specific high risk industries, if there is reasonable cause to suspect drug use and following a health and safety incident. The New Zealand Drug Foundation has suggested that the current regulations should be reformed. An examination of the relevant case law supports this position.

Key words: Workplace Drug Testing, Privacy, Impairment, Health and Safety, Civil Liberties 


\section{NOUVELLE-ZÉLANDE}

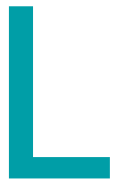

a question du dépistage des drogues sur le lieu de travail est très controversée dans la législation néo-zélandaise. En effet, il s'agit d'un conflit entre les droits et les responsabilités des uns et des autres, en l'occurrence le droit d'une personne de garder confidentielles ses décisions privées (y compris le droit d'utiliser des drogues à usage récréatif) par rapport à la responsabilité de l'employeur d'assurer un lieu de travail sûr.

Dans un article intitulé "Le dépistage des drogues n'est pas toujours la réponse », la New Zealand Drug Foundation estime que la réglementation actuelle concernant le dépistage des drogues sur le lieu de travail est défectueuse : "Le dépistage des drogues sur le lieu de travail n'améliore pas toujours la sécurité au travail, il peut être insuffisant pour détecter l'altération des facultés et il est très invasif (...). La réduction des déficiences sur le lieu de travail, plutôt que de la consommation de drogues, devrait être l'objectif principal. Cela ne peut se faire qu'à travers une gestion de la qualité, une culture consistant à signaler les risques en matière de santé et de sécurité, et un système encourageant les gens à s'exprimer s'ils remarquent un problème ou constatent qu'une personne n'est pas dans son état normal $»^{1}$.

En droit néo-zélandais, les dispositions relatives aux tests de dépistage sur le lieu de travail se trouvent dans les articles 30 à 46 du Health and Safety at Work Act (la loi sur la santé et la sécurité au travail)². Cette loi remplace le Health and Safety in Employment Act, qui régissait auparavant les tests de dépistage sur le lieu de travail ${ }^{3}$.

S'il peut sembler étrange que ces réglementations ne soient pas incluses dans la législation sur les relations de travail, cela est notamment dû au fait que la toxicomanie au travail est considérée comme un risque professionnel et a donc été regroupée avec d'autres risques en matière de santé et de sécurité 4 .

Après avoir analysé la jurisprudence néo-zélandaise (I) des propositions de réformes légales seront présentées (II).

1 «Drug testing isn't always the answer», New Zealand Drug Foundation: https://www.drugfoundation. org.nz/info/at-work/drug-testing/

2 Health and Safety Act, 2015, p. 30.

3 Health and Safety in Employment Act, 1992.

4 A. Green, J. Green et J. Heron, Laws of New Zealand, Liquor Law (édition en ligne) [282]. 


\section{I - LA JURISPRUDENCE NÉO-ZÉLANDAISE}

Chacune des affaires suivantes aborde un point de droit essentiel dans la réglementation actuelle du dépistage des drogues sur le lieu de travail en Nouvelle-Zélande. Cependant, deux questions principales sont récurrentes:

- d'une part, celle très controversée de la réglementation liée au lieu de travail,

- d'autre part, celle du des employeurs qui outrepassent fréquemment leurs pouvoirs en réalisant des tests de dépistage des drogues au travail.

\section{A - REFUS D'UN TEST DE DÉPISTAGE DE DROGUE : PARKER V. SILVER FERN FARMS LTD}

Parker était un boucher, salarié dans une usine de traitement de la viande appartenant à Silver Fern Farms. Alors que ses collègues et lui-même venaient d'arriver au travail, leur employeur a demandé la permission de fouiller leurs voitures. Parker, comme les autres salariés, ont consenti à cette perquisition.

Du cannabis a été retrouvé dans la voiture de Parker, ainsi que dans celles de plusieurs autres de ses collègues. Invité à rester sur le site mais sans se mettre au travail, Parker s'est plaint à son délégué syndical du stress qu'il ressentait et a quitté l'usine. Après consultation de son médecin, il a obtenu un arrêt de travail d'un mois ${ }^{5}$. Ses employeurs lui ont alors indiqué qu'il ne serait pas licencié pour avoir conservé du cannabis dans sa voiture, mais qu'il s'agissait là d'un dernier avertissement avant son renvoi.

En outre, ils ont également signifié à Parker qu'il l'obligerait à subir un test de dépistage de drogue avant de pouvoir reprendre le travail, signifiant de la sorte qu'il était légitime de lui faire passer le test de dépistage dans la mesure où le cannabis découvert dans sa voiture constituait un motif raisonnable de soupçonner qu'il consommait des drogues. Parker a refusé de subir le test de dépistage. Son employeur a alors mis fin à son contrat avec Silver Fern Farms ${ }^{6}$.

Dans cette affaire, il a été jugé que le fait que Silver Fern Farms demande à Parker de se soumettre à un test de dépistage était justifié, car cela protégeait la santé et la sécurité du salarié lui-même et de ses collègues. Il a également été jugé que Parker n'avait pas de raison valable de refuser de passer le test de dépistage. En vertu de son contrat de travail, Parker s'était engagé à se conformer à la politique de Silver Fern Farm en matière de drogue et d'alcool, qui prévoyait des tests de dépistage en cas de soupçons raisonnables sur la consommation de drogue.

Par ailleurs, le refus de passer un test de dépistage était considéré comme une faute grave dans le cadre de la politique de l'entreprise en matière de drogue et d'alcool. Comme Parker en était déjà à son dernier avertissement en raison du cannabis trouvé dans sa voiture, cet acte supplémentaire - constituant une faute grave - suffisait à justifier son licenciement par Silver Fern Farms ${ }^{7}$.

5 Parker v. Silver Fern Farms Ltd [2009] ERNZ 301.

6 Ibid.

7 Ibid. 


\section{NOUVELLE-ZÉLANDE}

L'arrêt du tribunal du travail contient le passage suivant, qui a eu une grande influence sur les affaires ultérieures liées au dépistage des drogues. La Cour a ainsi déclaré : "Les régimes de dépistage de drogue sur des salariés empiètent considérablement sur les droits et libertés individuels. Non seulement les politiques, comme leur application, doivent répondre aux critères juridiques et constituer des instructions licites et raisonnables données aux salariés, mais lorsque celles-ci sont contenues dans des politiques promulguées par l'employeur, elles doivent être interprétées et appliquées strictement ${ }^{8}$.

Cette affaire a eu un impact important pour plusieurs raisons :

- d'une part, elle a confirmé que le dépistage des drogues doit figurer dans la politique de l'employeur en matière de drogue et d'alcool si celui-ci souhaite avoir recours à des tests ; - d'autre part, elle a démontré que la possession de drogue pouvait être un motif raisonnable de soupçonner qu'un salarié en consomme lui-même. Plus important encore, elle a établi que le refus de se soumettre, sans raison valable, à un test de dépistage pouvait constituer une faute grave?

\section{B - Suffisance du motif : Sim v. Carter Holt Harvey Ltd et Hooper $v$. Coca-Cola AMATIL}

\section{1 - Sim v. Carter Holt Harvey Ltd}

Carter Holt Harvey exploitait une scierie à Eves Valley. Suite à la découverte de deux plants de cannabis poussant sur le site de la scierie, Carter Holt Harvey a demandé aux 190 salariés du site de se soumettre à un test de dépistage de drogue. Le test de dépistage effectué sur l'un de ces salariés, Sim, a donné un résultat négatif. En outre, il n'existait aucune preuve indiquant que Sim avait planté le cannabis ${ }^{10}$.

Carter Holt Harvey a soutenu que la découverte des plantes lui donnait un motif raisonnable de faire passer un test de dépistage à tous ses salariés. En l'occurrence, le libellé exact de la clause relative au dépistage dans sa politique interne en matière d'alcool et de drogues indiquait qu'il y aurait un motif raisonnable de faire un test de dépistage si "l'apparence, les actions ou le comportement d'un salarié suggèrent qu'il peut être sous l'influence de la drogue ou de l'alcool ${ }^{11}$.

Or, I'Employment Relations Authority (ERA) a estimé qu'aucun des salariés du site n'avait présenté les caractéristiques indiquant une consommation des drogues mentionnées dans la politique interne de l'entreprise.

Par ailleurs, I'ERA a jugé que le critère du motif raisonnable ne pouvait être appliqué qu'à un individu, et non à l'ensemble du personnel ${ }^{12}$. Le tribunal a déclaré : "Cela signifie qu'avant qu'un test ne se produise, il doit y avoir un motif raisonnable pour que le défendeur subisse ce test. Il doit y avoir un lien entre le motif raisonnable et le test, car le test est considéré comme une manière de corroborer le motif raisonnable $»^{13}$.

8 Parker v. Silver Fern Farms Ltd [2009] ERNZ 301, op. cit.

9 Ibid.

10 Sim v. Carter Holt Harvey Ltd [2014] NZERA 336.

11 lbid.

12 lbid.

13 lbid. 
Cette affaire a établi les limites de ce qui constitue un motif raisonnable pour le dépistage des drogues au travail, ce dernier ne pouvant être réalisé que si une personne fait preuve d'un comportement qui suggère effectivement une consommation de drogue ${ }^{14}$.

\section{2 - Hooper v. Coca-Cola Amatil}

Hooper a été invité par son employeur à passer un test de dépistage de drogue au travail. Le résultat indiquait une consommation de cannabis en grande quantité ${ }^{15}$. En vertu de la convention collective dont dépendait Hooper, l'employeur pouvait effectuer un test de dépistage s'il existait des motifs raisonnables de soupçonner les salariés de consommer de la drogue. De ce fait, le dépistage aléatoire des drogues n'était pas prévu par la convention collective $^{16}$.

Lors de la réunion du conseil de discipline qui s'est tenue entre Hooper et l'entreprise, l'intéressé a été informé qu'un enquêteur privé, engagé par l'entreprise, l'avait vu fumer du cannabis et qu'un dénonciateur anonyme l'avait également accusé d'en consommer. Cette information n'avait pas été préalablement communiquée à Hooper. Il fut finalement licencié ${ }^{17}$.

Le tribunal du travail a jugé que l'employeur de Hopper n'avait pas de motif raisonnable de le tester, ce que la société a elle-même reconnu par la suite. Fait important, le tribunal a estimé que même si Hooper avait échoué au test de dépistage de drogues, cela ne signifiait pas que l'entreprise avait le droit de lui faire subir un test sans soupçon raisonnable.

En outre, la Cour a jugé que la société avait commis une erreur en ne fournissant pas à Hooper les éléments prouvant sa consommation de drogue préalablement à la réunion du conseil de discipline ${ }^{18}$.

\section{C - Les CONDitions du dÉPISTAge de dRogues: NZ AmAlgamated Engineering Printing \& Manufacturing Union Inc v. Air NeW ZEALAND LTD}

Six syndicats ont enjoint Air New Zealand de ne pas effectuer des tests de dépistage de drogue et d'alcool sur ses salariés. A l'époque, aucun des six syndicats n'avait conclu avec Air New Zealand de convention collective prévoyant des dispositions sur les tests de dépistage de drogues et d'alcool ${ }^{19}$.

Dans sa décision, le tribunal du travail a établi des prescriptions dans plusieurs domaines clés du dépistage des drogues. Plus important encore, le tribunal a jugé que le dépistage aléatoire des drogues ne pouvait être réalisé que dans des professions "sensibles sur le plan de la sécurité », comme par exemple celles impliquant la conduite de gros véhicules, l'industrie ou la récolte du bois. Ces tests doivent être effectués de manière spécifique

14 Ibid.

15 Hooper v. Coca-Cola Amatil (NZ) Ltd (2012) 9 NZELR 523.

16 Ibid.

17 Ibid.

18 lbid.

19 NZ Amalgamated Engineering Printing \& Manufacturing Union Inc v Air New Zealand Ltd [2004] 1 ERNZ 614. 


\section{NOUVELLE-ZÉLANDE}

par une entité indépendante, telle qu'une société de dépistage de drogues. L'employeur doit également consulter les experts scientifiques appropriés pour interpréter à nouveau les résultats, ce qui peut être effectué par une entreprise spécialisée dans le dépistage de drogues.

Cela impliquait pour Air NZ d'utiliser le dépistage aléatoire de drogues que pour les salariés travaillant dans certains domaines, et non pas pour tout son personne ${ }^{20}$.

La décision du tribunal d'autoriser le dépistage aléatoire des drogues n'a pas été prise à la légère. Cette déclaration faisait référence au fait qu'une telle politique soulevait de graves problèmes de confidentialité. La Cour a ainsi justifié sa décision de la manière suivante: "La preuve que les tests aléatoires ont un effet dissuasif nous incite à considérer que dans les secteurs sensibles sur le plan de la sécurité, dans lesquels les conséquences peuvent être catastrophiques, l'objection à l'utilisation de méthodes intrusives de surveillance dans le but d'éliminer un danger reconnu doit passer après les considérations de sécurité. Ces facteurs ont priorité sur les problèmes de confidentialité ${ }^{21}$.

Le tribunal a également donné des indications détaillées sur l'élaboration des politiques internes en matière de drogue et d'alcool, signalant la nécessité de consulter les salariés et les syndicats. De ce fait, le consentement des salariés doit être sollicité avant le test et, dans certains cas, le fait de refuser de passer un test de dépistage revient à un refus d'obéir à des instructions légitimes et raisonnables, susceptible de constituer un motif de licenciement. Cependant, chaque cas devrait être tranché individuellement ${ }^{22}$.

Dans son jugement, le tribunal a par ailleurs noté qu'à l'exception de l'alcool, un test dont le résultat indique la présence de drogues ne signifie pas automatiquement que les facultés du sujet étaient altérées au moment du contrôle. Il a également jugé que l'objectif principal d'un programme de dépistage des drogues devait être la sensibilisation des salariés et la prévention de la toxicomanie. De plus, la réadaptation des toxicomanes devrait être la principale solution pour les salariés dont le test est positif. Ces points seront pertinents lorsqu'il s'agira d'étudier la question des réformes de la réglementation sur le dépistage des drogues ${ }^{23}$.

Cette affaire constitue l'élément central du droit applicable sur les drogues en Nouvelle-Zélande, la conclusion la plus importante étant que le dépistage aléatoire des drogues ne peut être utilisé que pour certaines professions ou industries jugées « sensibles sur le plan de la sécurité $»^{24}$.

20 Ibid.

21 NZ Amalgamated Engineering Printing \& Manufacturing Union Inc v. Air New Zealand Ltd, op. cit.

$22 \mathrm{Ibid}$.

23 Ibid.

$24 \mathrm{lbid}$. 


\section{D - LA DÉFINITION DE LA NOTION « SENSIBLE SUR LE PLAN DE LA SÉCURITÉ » : ELECTRICAL UNION 2001 INC V. Mighty RIVER POWER LTD}

La notion - «sensible sur le plan de la sécurité »- a été clarifiée dans une action intentée par un syndicat contre la société d'énergie Mighty River Power ${ }^{25}$. Cette société avait mis en place une politique en matière de drogue et d'alcool exigeant que tout travailleur employé dans un "domaine ou poste sensible sur le plan de la sécurité " soit soumis à des tests de dépistage aléatoires ${ }^{26}$.

Dans sa politique relative aux drogues et à l'alcool, la société l'avait ainsi défini en ces termes: "Tout domaine ou poste, dans lequel le fait de ne pas remplir correctement les tâches concernées par ces fonctions exposerait la personne, ou d'autres personnes, à un risque de blessure, préjudice, préjudice grave ou de dommages à la propriété, à l'entreprise ou aux l'équipements ${ }^{27}$.

Travailleur syndiqué, Cowell occupait un poste de technicien de production géothermique dans une centrale géothermique exploitée par Mighty River Power. Ses fonctions et son lieu de travail étaient définis comme "sensibles sur le plan de la sécurité " selon la politique en matière de drogue et d'alcool de Mighty River Power. Il lui a été demandé de passer un test de dépistage de drogues sur le lieu de travail. Refusant de le faire, Cowell a été suspendu ${ }^{28}$.

Dans cette affaire NZ Amalgamated Engineering Printing \& Manufacturing Union Inc v. Air New Zealand Ltd, I'ERA a estimé que la décision octroyait le droit à Mighty River Power de tester aléatoirement les salariés affectés à des postes ou dans des lieux « sensibles sur le plan de la sécurité ». Elle a jugé que la décision de classer certains postes ou lieux comme étant « sensibles » relevait des prérogatives de Mighty River Power, puisque c'est aussi à la société qu'il incombait de s'assurer du respect des normes réglementaires en matière de santé et de sécurité ${ }^{2}$.

Le droit de faire subir des tests de dépistage aléatoires aux salariés affectés à des postes ou dans des sites " sensibles sur le plan de la sécurité » représente déjà un pouvoir important pour les employeurs sur leurs salariés. En permettant aux employeurs de définir également les postes et les sites "sensibles", cette décision élargit considérablement les pouvoirs de l'employeur ${ }^{30}$.

25 Electrical Union 2001 Inc v Mighty River Power Ltd [2013] NZERA 117.

$26 \mathrm{lbid}$.

27 Ibid.

28 Electrical Union 2001 Inc v Mighty River Power Ltd [2013] NZERA 117, op. cit.

29 Ibid.

$30 \mathrm{lbid}$. 


\section{NOUVELLE-ZÉLANDE}

\section{E - PolitiQue d'ENTREPRISE SUR LES DROgUeS ET L'ALCOOL: MARITIME UNION OF NEW ZEALAND INC V. TLNZ LTD}

Cette affaire visait à déterminer dans quelle mesure une entreprise peut appliquer une politique interne sur les drogues et l'alcool, si ses salariés et leurs représentants syndicaux n'ont pas accepté sa mise en œuvre ${ }^{31}$. En l'espèce, l'entreprise TLNZ Ltd avait élaboré une politique en matière de drogue et d'alcool, en concertation avec ses salariés et leur syndicat.

Cependant, cette politique interne contenait des dispositions permettant à l'entreprise de contrôler l'haleine et l'urine des salariés afin de détecter une éventuelle consommation d'alcool ou de drogues. En désaccord avec ces dispositions, le syndicat a soutenu que TLNZ ne pouvait pas appliquer ce règlement sans son consentement ${ }^{32}$.

Le tribunal du travail a jugé que les employeurs des secteurs « sensibles sur le plan de la sécurité » pouvaient imposer leurs propres politiques en matière de drogue et d'alcool à leurs salariés, ceci donc sans leur consentement. II n'appartenait pas aux tribunaux de juger du caractère raisonnable de ces politiques, ou des méthodes de dépistage susceptibles d'être employées, à moins qu'une action n'ait été spécifiquement introduite à cet effet devant un tribunal ${ }^{33}$.

Point important s'agissant de la réforme de la réglementation sur le dépistage des drogues: la Cour a également déclaré que l'employeur était libre de choisir le test d'urine, au lieu du test de salive. A cet égard, il a jugé légitime l'objectif de l'employeur qui utilise le test d'urine pour identifier les salariés susceptibles d'être sous l'influence de la drogue ou de l'alcool au travail. Par ailleurs, TLNZ n'avait pas besoin de prouver que les facultés du salarié étaient altérées au moment du test ${ }^{34}$.

Ce raisonnement de la Cour érode considérablement le droit des travailleurs quant au respect de leur vie privée. En effet, ces moyens de dépistage ne semblent pas utilisés pour déterminer si les capacités du travailleur sont altérées au moment du test, mais visent plutôt à déterminer si un travailleur est un consommateur de drogues durant ses loisirs. Bien qu'un employeur puisse justifier ces méthodes de dépistage en invoquant l'argument de l'amélioration de la santé et de la sécurité, il s'agit néanmoins indéniablement d'une ingérence importante de l'employeur dans les activités du salarié en dehors du travail ${ }^{35}$.

\section{F - CONSOMMATION DE DROGUES EN DEHORS DU TRAVAIL ET TESTS DE SALIVE : HAYLLAR V. THE GOODTIME FOOD COMPANY LTD}

Cette affaire met en évidence une question centrale : celle du droit du salarié à l'autonomie dans ses choix de vie personnels (y compris la consommation ou non de drogues récréatives) et la responsabilité de l'employeur de maintenir la santé et la sécurité

31 Maritime Union of New Zealand Inc v TLNZ Ltd (2007) 5 NZELR 87.

32 lbid.

33 lbid.

$34 \mathrm{lbid}$.

35 Maritime Union of New Zealand Inc v TLNZ Ltd (2007) 5 NZELR 87, op. cit. 
au travail ${ }^{36}$. Cette question jurisprudentielle est intimement liée aux caractéristiques techniques des tests d'urine par rapport aux tests de salive ${ }^{37}$.

Deux travailleurs, Hayllar et Matene, s'étaient querellés avec leur supérieur hiérarchique. L'entreprise qui les employait, Goodtime Food Company, avait mis en place une politique de dépistage des drogues en cas de motif raisonnable. En l'espèce, l'entreprise a estimé que cette dispute constituait un motif raisonnable de faire subir un test de dépistage aux deux hommes. Or les tests subis par les deux salariés ont révélé la présence d'acide THC dans leur urine. Il leur fut donc proposé de continuer à travailler pour l'entreprise, à condition de suivre un programme de désintoxication. Hayllar et Matene ont accepté et ont poursuivi leurs activités au sein de Goodtime ${ }^{38}$.

Au cours des semaines suivantes, Hayllar fut victime d'un léger accident du travail et Goodtime lui ordonna de passer un deuxième test de dépistage. En outre, un cadre de l'entreprise avait remarqué, lors d'une sortie entre collaborateurs, que Matene dégageait une odeur de cannabis et ainsi, il lui a également été demandé de passer un deuxième test de dépistage. Les second tests subis par Hayllar et Matene ont indiqué la présence d'acide THC dans leurs urines ${ }^{39}$.

L'affaire a été portée devant le tribunal du travail. Soutenant que les tests d'urine pour le dépistage du cannabis n'étaient pas destinés à mesurer l'altération des facultés au moment du contrôle, mais seulement le fait que le sujet testé avait consommé du cannabis au cours des derniers jours, l'avocat de Hayllar a considéré qu'il était injuste de les utiliser comme base pour des mesures disciplinaires. Le dépistage des drogues par l'urine dictait effectivement ce que Hayllar pouvait faire en dehors du lieu de travail, sans indiquer si ces capacités étaient altérées dans son travail. Par conséquent, il conviendrait de réaliser un test de salive pour dépister la consommation de cannabis, ce qui montrerait avec beaucoup plus de précision si les facultés du sujet testé étaient amoindries au moment du contrôle ${ }^{40}$.

Il existe une norme commune pour les tests de dépistage de drogue dans l'urine en Australie et en Nouvelle-Zélande. En conséquence, le tribunal du travail a estimé qu'une décision australienne - dans l'affaire Endeavour Energy v. Communications, Electrical, Electronic, Energy, Information, Postal, Plumbing and Allied Services Union of Australia " s'appliquait également » en Nouvelle-Zélande : "L'employeur a le droit légitime (et même l'obligation) d'essayer d'éliminer le risque que les salariés viennent au travail sous l'emprise des drogues ou de l'alcool, au point de constituer un risque pour la santé ou la sécurité. Par-delà, l'employeur n'a pas le droit d'indiquer à son salarié quels sont les drogues ou les alcools qu'il peut consommer sur son temps libre. Ce serait en effet injuste et déraisonnable» ${ }^{41}$.

36 Hayllar v The Goodtime Food Company Ltd (2012) 10 NZELR 455.

$37 \mathrm{lbid}$.

$38 \mathrm{lbid}$.

39 Ibid.

40 Ibid.

41 Endeavour Energy v. Communications, Electrical, Electronic, Energy, Information, Postal, Plumbing and Allied Services Union of Australia (2016) 244 FCR 178. 


\section{NOUVELLE-ZÉLANDE}

Finalement, le tribunal du travail a donc jugé que l'entreprise Goodtime s'était comportée de manière inacceptable en n'informant pas précisément Hayllar et Matene que leur échec au premier test de dépistage de drogues était grave, et que leur simple participation au programme de réadaptation n'avait pas totalement clos le problème. II a également jugé qu'ils n'auraient pas dû subir le deuxième test de dépistage de drogues ${ }^{42}$.

Malheureusement, le tribunal du travail n'a pas apporté beaucoup d'explications sur la question des tests d'urine par rapport aux tests de salive. Une décision claire de la Cour en faveur de l'analyse d'urine aurait ainsi permis d'indiquer que les obligations des employeurs en matière de santé et de sécurité l'emportaient sur la vie personnelle des travailleurs. A l'inverse, la préconisation des tests de salive aurait démontré le contraire ${ }^{43}$.

\section{II - LES RÉFORMES POSSIBLES}

Il existe un certain nombre de réformes simples à mettre en œuvre qui pourraient réduire considérablement le caractère invasif du dépistage des drogues au travail pour la vie privée d'un salarié, tout en maintenant des normes élevées de santé et de sécurité au travail.

\section{A - LA DÉSIGNATION DES POSTES, INDUSTRIES ET LIEUX « SENSIBLES SUR LE PLAN DE LA SÉCURITÉ ॥}

Il serait pertinent que la législation revienne sur la décision adoptée dans l'affaire Electrical Union 2001 Inc v. Mighty River Power Ltd. En effet, en permettant aux employeurs de déterminer les professions et les sites "sensibles sur le plan de la sécurité ", cette décision les incite à attribuer trop aisément cette qualification, leur permettant ainsi de réaliser des tests aléatoires sur un plus grand nombre de salariés. Une liste officielle des professions, des industries et des emplacements "sensibles " devrait être officiellement créée et tenue à jour par un organisme tiers, qui pourrait aussi bien être une organisation gouvernementale ou une entreprise privée ${ }^{44}$.

\section{B - L'INTRODUCTION D'UN NOUVEAU CRITÈRE : L'ALTÉRATION DES FACULTÉS AU MOMENT DU TEST}

Force est de constater que, dans l'affaire Maritime Union of New Zealand Inc v. TLNZ Ltd, la décision rendue est injuste ${ }^{45}$ et devrait également être neutralisée par un texte législatif. Ce jugement autorise explicitement les employeurs à effectuer des tests de dépistage sur le lieu de travail, afin de déterminer quels salariés consomment des drogues, et non pas quels salariés ont des capacités altérées par les drogues, dans leur travail.

42 Hayllar v. The Goodtime Food Company Ltd, op. cit.

43 lbid.

44 Electrical Union 2001 Inc v. Mighty River Power Ltd [2013] NZERA 117, op. cit.

45 Maritime Union of New Zealand Inc v. TLNZ Ltd (2007) 5 NZELR 87, op. cit. 
Bien que la justification invoquée est que cette décision permettait aux employeurs d'identifier ceux des salariés qui représentaient un risque en matière de santé et de sécurité, ce raisonnement paraît insatisfaisant pour plusieurs raisons. Premièrement, même si un salarié consomme des drogues à usage récréatif en dehors du travail, cela ne signifie pas que ses facultés au travail en seront affectées. Plus important encore, ce raisonnement constitue une discrimination intrinsèque vis-à-vis de certains salariés, en raison de leurs choix personnels en dehors du travail.

L'affaire NZ Amalgamated Engineering Printing \& Manufacturing Union Inc v. Air New Zealand $L t d$ soutient ce raisonnement ${ }^{46}$. En l'espèce, le tribunal a convenu qu'un test de dépistage positif ne signifiait pas que les facultés du sujet étaient altérées au moment du test.

En outre, il a déclaré que le principal objectif du dépistage des drogues sur le lieu de travail était d'informer les salariés sur la toxicomanie. Cette démonstration est très différente de l'objectif qui consiste à identifier les salariés présentant un risque en matière de santé et de sécurité, invoqué par les employeurs dans l'affaire Maritime Union of New Zealand Inc v TLNZ Ltd.

De même, dans l'affaire Hayllar v. The Goodtime Food Company Ltd, le tribunal a soutenu l'allégation selon laquelle un employeur n'avait pas le droit de décider quelles drogues un salarié pouvait consommer en dehors du travail ${ }^{47}$. Cette décision s'oppose totalement à celle qui a été adoptée dans l'affaire Maritime Union of New Zealand Inc v. $T L N Z$ Ltd. En l'espèce, la déclaration du tribunal dans l'affaire Hayllar peut logiquement être considérée comme favorable à la seule mesure de l'altération des facultés au moment du test ${ }^{48}$. En effet, si l'employeur n'a pas le droit de décider quelles sont les drogues qu'un salarié peut consommer en dehors du travail, il ne devrait pas être en mesure de rechercher celles qu'un salarié aurait pu consommer hors des heures de travail, mais uniquement celles qui altèrent ses facultés au travail.

D'un point de vue technique, cela signifie que l'utilisation des tests d'urine pour le dépistage sur le lieu de travail devrait être purement et simplement supprimée. Or actuellement, l'utilisation des tests de salive comme méthode de dépistage n'est pas approuvée ${ }^{49}$. Il serait bon qu'elle le soit, comme c'est le cas en Australie ${ }^{50}$. En revanche, les tests sanguins sont disponibles en Nouvelle-Zélande et donnent un ensemble de résultats beaucoup plus complexe que les tests d'urine ${ }^{51}$. Cela permet de déterminer plus facilement si les facultés d'un salarié sont effectivement altérées au moment du test et s'il présente par conséquent un risque en matière de santé et de sécurité.

46 NZ Amalgamated Engineering Printing \& Manufacturing Union Inc v. Air New Zealand Ltd [2004] 1 ERNZ 614, op. cit.

47 Hayllar v. The Goodtime Food Company Ltd (2012) 10 NZELR 455, op. cit.

48 lbid.

49 «Drug testing isn't always the answer», New Zealand Drug Foundation, op. cit.

50 lbid.

51 lbid. 


\section{NOUVELLE-ZÉLANDE}

Dès lors, le dépistage des drogues sur le lieu de travail ne devrait être utilisé que pour déterminer si un salarié est atteint d'une déficience au moment du contrôle. II ne doit pas être destiné à découvrir si un salarié consomme des drogues à usage récréatif en dehors du travail.

En conclusion, il est important de respecter le droit des travailleurs de consommer des drogues à usage récréatif en dehors du travail, sans que l'employeur en soit informé. Ceci fait partie intégrante du droit fondamental à la vie privée.

La jurisprudence démontre qu'en Nouvelle-Zélande, la réglementation sur le dépistage des drogues sur le lieu de travail est beaucoup trop intrusive et devrait donc être réformée. 